\title{
Biomedical imaging research opportunities workshop IV: A white paper
}

William R. Hendee ${ }^{\text {a) }}$

Medical College of Wisconsin, 8701 Watertown Plank Road, Milwaukee, Wisconsin 53226

Filip Banovac

Georgetown University Hospital, Washington, DC 20007

Paul L. Carson

The University of Michigan Medical Center, Ann Arbor, Michigan 48109

Ralph A. DeFronzo

University of Texas Health Sciences Center, San Antonio, Texas 78201

William C. Eckelman

Molecular Tracer, LLD, Bethesda, Maryland 20892

Gary D. Fullerton

University of Texas Health Sciences Center, San Antonio, Texas 78201

Steven M. Larson

Memorial Sloan-Kettering Cancer Center, New York, New York 10021

Gordon McLennan

Indiana University, Indianapolis, Indiana 46202

Michael J. Welch

Mallinckrodt Institute, St. Louis, Missouri 63110

(Received 26 April 2006; revised 22 June 2006; accepted for publication 12 July 2006; published 26 January 2007)

The Fourth Biomedical Imaging Research Opportunities Workshop (BIROW IV) was held on February 24-25, 2006, in North Bethesda, MD. The workshop focused on opportunities for research and development in four areas of imaging: imaging of rodent models; imaging in drug development; imaging of chronic metabolic disease: diabetes; and image guided intervention in the fourth dimension-time. These topics were examined by four keynote speakers in plenary sessions and then discussed in breakout sessions devoted to identifying research opportunities and challenges in the individual topics. This paper synthesizes these discussions into a strategy for future research directions in biomedical imaging. (C) 2007 American Association of Physicists in Medicine. [DOI: 10.1118/1.2405838]

Key words: rodents, drug development, chronic disease, diabetes, image-guided therapy, imaging technology, imaging research

\section{OVERVIEW}

The Fourth Biomedical Imaging Research Opportunities Workshop (BIROW IV) was held on February 24-25 in North Bethesda, Maryland. BIROW IV was sponsored by the Academy of Radiology Research (ARR), American Association of Physicists in Medicine (AAPM), American Institute for Medical and Biological Engineering (AIMBE), Biomedical Engineering Society (BMES), and the Radiological Society of North America (RSNA). Nineteen other medical imaging societies served as participating organizations in the meeting. The purpose of BIROW IV (as of BIROWs I, II and III, which were held in 2003, 2004, and 2005) ${ }^{1-3}$ was to identify and characterize opportunities for scientific research and engineering development in biomedical imaging.

BIROW IV focused on four imaging areas that offer a spectrum of opportunities for scientific research and engineering development:

(1) Imaging of rodent models;
(2) Imaging in drug development;

(3) Imaging of chronic metabolic disease: Diabetes;

(4) Image-guided intervention in the fourth dimension-time.

Each area was addressed in a plenary session in which four speakers presented their perspectives on the subject and the research opportunities and challenges it presents, followed by audience discussion. Each topic was then the focus of discussion at one of four simultaneous breakout sessions. Each breakout session presented a forum for the discussion of research opportunities in the area from four perspectives related to the objectives of the Roadmap Program of the National Institutes of Health (NIH). ${ }^{4}$ These four perspectives were:

(1) Does it deepen understanding of fundamental biology?

(2) Does it promote collaboration of multidisciplinary teams?

(3) Does it reshape clinical research and promote discovery?

(4) Does it improve people's health? 
Breakout participants were also asked to identify challenges to the realization of the research opportunities. The four questions for this section of the sessions were:

(1) What are the scientific challenges?

(2) What are the primary obstacles to development?

(3) What are the critical technologies that are lacking?

(4) What are the impediments to translating the opportunity to improved health?

The plenary and breakout sessions yielded a wealth of information that has been synthesized and edited into the findings and recommendations presented in this paper.

\section{IMAGING OF RODENT MODELS}

Rodents serve as models of human disease and as test vehicles for guiding and evaluating novel diagnostic and therapeutic tools to improve the identification and intervention of disease. Imaging of rodent models provides insight into the molecular mechanisms of health and disease, and enables researchers to monitor the evolution of disease over time and in response to various interventions. ${ }^{5-24}$

\section{RODENT IMAGING: OPPORTUNITIES}

Rodent imaging enables qualitative and quantitative assessment of normal and abnormal biological processes, including tissue functions such as apoptosis, senescence, stasis, dormancy, necrosis, and fibrosis, among others. Imaging enables these processes to be tracked over time without requiring the periodic sacrifice of experimental animals. Developmental abnormalities, for example, can best be evaluated by imaging temporal changes in the same group of animals over an extended time period. Further, imaging studies are often substantially less expensive than those that require the periodic sacrifice of animals. New knowledge in rodent genetics has enabled the development of genetically-mutated rats with specific disease characteristics, leading to dramatic growth in the number of human diseases able to be emulated in rodent models.

New rodent imaging tools make it possible to research new questions about fundamental biology. Answers to these questions often lead to an identified need for new imaging tools to pursue further what has been learned. That is, the link between new tools and new applications is an iterative cycle in which each reinforces the other, thereby advancing the frontiers of new knowledge in human health and disease through research with rodents. This research is multidisciplinary in nature, involving basic and computer scientists, engineers, mathematicians, and physicians working together in the use of rodent imaging tools to acquire new knowledge.

Rodent imaging sits at the crossroads between basic research on rodent models of human disease and the evaluation of treatment methods for human disease using rodent models. In many cases rodent imaging is the pathway to understanding the linkage between the phenotypic expression of disease and the underlying genetic cause of the disease. Im- aging of rodent models accelerates the process of drug discovery by enabling longitudinal studies of drug effectiveness with internal controls.

Rodent imaging methods have the potential to bring new therapies (drugs) to market sooner, with reduced development costs, by providing more efficient pathways for preclinical evaluation of the therapies. Potential contributions include: (1) confirmation that the drug is delivered to and interacts with its intended target; (2) measurement and monitoring over time of the pharmacokinetics, targeting efficiency, pharmacodynamics, and biodistribution of the drug; (3) characterization of the target tissue; and (4) identification of subpopulations of cells within the target that respond differently to the drug. Rodent imaging can also reduce the time and cost associated with evaluating nondrug therapies such as tumor-ablation techniques, radiation treatments, and genetic manipulation of tissues. Finally, technologies for rodent imaging can sometimes be adapted for human application.

\section{RODENT IMAGING: CHALLENGES}

Rodent imaging research faces several challenges, including the need for: improved quantitative tools and procedures; higher frame rates and throughput; greater contrast, spatial, and temporal resolution; improved temporal and spatial registration of physiological and molecular data with images; higher sensitivity and, in cases where ionizing radiation is employed, reduced dose; integrated instrumentation to eliminate sequential multi-modality imaging studies that are difficult and time-consuming; standardized imaging protocols to facilitate multi-institutional collaboration; fast, simple, and low-cost rodent imaging systems; enhanced ability to distinguish effects of a therapy (drug) from the effects of the imaging technique (ionizing radiation), anesthesia procedure, or contrast agent employed.

There is not a centralized source of interest in and funding for development of rodent imaging systems. The emphasis at the NIH on hypothesis-driven research compromises the competitiveness of funding applications that propose the development of new imaging tools and techniques. The Department of Energy, a substantial supporter of imaging research in the past, is gradually phasing out its support of such research. The private sector has made only a modest investment in rodent imaging systems and research. One suggestion is the co-location of rodent housing and imaging facilities in selected locations across the country, enabling investigators to achieve cost- and facility-sharing.

There is an opportunity for further technical development in several rodent imaging technologies. For example, there is currently a need for: high frequency ultrasound transducer arrays that provide simultaneous transmission and reception of ultrasound signals from tissues; rodent-size x-ray computed tomographic units that provide higher speed and contrast resolution than that currently available with rodent CT systems; more efficient $\mathrm{x}$-ray systems that permit dose reduction to rodents and a measure of the actual dose delivered to the detectors and the animals; magnetic resonance imaging coils for small animal imaging; imaging systems that are fast 
enough to permit imaging of nonanesthetized animals; further evolution of methods such as magnetic resonance imaging and spectroscopy that provide quantitative information about tissues in addition to images; new imaging methods that do not employ ionizing radiation, including techniques such as optical imaging and in vivo optical microscopy; development of high-specificity radioactive tracers and contrast agents for genomic and proteomic targets.

Rodent imaging is a young and immature field. Its contributions to understanding human health and disease and to improved methods for designing and evaluating diagnostic and treatment techniques are just beginning to be appreciated. Equipment for rodent imaging is still in its infancy, and many areas for improvement can be identified. Finally, additional animal models for human disease are needed, especially those for diseases other than cancer.

\section{IMAGING IN DRUG DEVELOPMENT}

More often than not, the effectiveness of a new drug is determined by a blinded, randomized, and controlled clinical trial in which symptoms, survival, and specific surrogate measures are compared between investigational and control patient groups. This technique is effective and will probably not be replaced in the foreseeable future as the final step (in vitro cell culture studies, animal model studies, and human clinical trials) in the approval of a drug for marketing and production. However, clinical trials are time-consuming, expensive, and highly inefficient in eliminating drugs that prove to be ineffective.

If it were effective in separating promising from nonpromising drugs, a preliminary step before clinical trials would accelerate the process of bringing drugs to market because financial, personnel, and patient resources could be devoted to just the promising drugs. Such a step would also be financially advantageous, because money would not be spent on clinical trials of drugs that ultimately prove to be physiologically- or cost-ineffective. The current interest in imaging methods as a mechanism to accomplish this separation process reflects an evolution in the understanding of disease from the level of anatomy, physiology, and histology to the cellular, molecular, and genetic levels. This evolution has occurred as a result of knowledge gained through systems biology. Imaging in drug development promises to result in smaller, less expensive clinical trials with fewer patients; earlier go/no go decisions on compounds; faster regulatory approval; and shorter time-to-market of drugs. $^{25-37}$

\section{IMAGING IN DRUG DEVELOPMENT: OPPORTUNITIES}

Sophisticated imaging technologies provide images in registration with quantitative physiological and functional data. They can yield spatial, temporal, and distributional information that may reflect the ability of drugs to concentrate and effect changes in specific molecular targets. This ap- proach can translate receptor-enzyme studies across disciplines and can validate in humans the information gained in preclinical studies of a drug.

To be useful in drug evaluation studies, imaging data must be held to the same rigorous standards as all data collected during clinical trials involving patients. Imaging procedures should meet agreed-upon standards for image acquisition, image quality, quantitative measurements, and data analysis. Further, quality assurance procedures should be instituted for human observer studies, interpretations, and measurements.

Use of imaging to validate the drug-delivery process and to evaluate the effectiveness of drugs is a multidisciplinary effort. Imaging physicists, engineers, bioinformaticians, computer modelers, pharmacologists, physicians, and statisticians are required to make the process successful. Such a collaboration requires substantial cross-training and an enhanced ability to communicate across disciplines and terminologies. Workshops should be conducted to explore the potential of imaging in drug development, and to identify ways to enhance interdisciplinary communication and collaboration. Identification of useful biomarkers for the effectiveness of specific drugs should be a focus of some of these workshops.

Imaging in drug development could yield databases of information used to classify patients according to their responsiveness to specific drugs. Creation of consortia of academic, industry, and government representatives interested in specific problems (e.g., imaging protocols, optimum biomarkers, targeted agents, quantitative assessment) would help expedite the deployment of imaging methods in drug development. These groups could share data for metanalysis directed at determining a drug's therapeutic and costeffectiveness. The shared database should be searchable to maximize its potential application to future drug development.

\section{IMAGING IN DRUG DEVELOPMENT: CHALLENGES}

Key surrogate markers must be identified for specific drugs so that their biodistribution and effectiveness can be determined. In the field of imaging, appropriate tracers and contrast agents must be designed to yield data important to the development and biodistribution of drugs that are effective in addressing the conditions of patient subpopulations. A partnership is needed between the pharmaceutical industry and the scientific community to yield drugs tagged with tracers to reveal the biodistribution of the drugs.

The mechanisms of action of specific drugs should be better understood at the molecular level, so that the right drugs can be developed for the right targets and for the right diseases. Currently there is little sense of urgency to accomplish these goals, a situation that must be corrected if drug development and deployment are to be made more timely and cost-effective. A networked infrastructure to support collaborative efforts to expedite drug development and deployment would contribute to a greater sense of urgency and enhanced efficiency in getting effective drugs to market in a more cost-effective manner. This infrastructure should in- 
clude consortia of academia, industry, and government agencies, especially regulatory agencies responsible for drug approval.

The usefulness of imaging in drug development depends on consensus about the instrumentation and data-acquisition methods to be used; the presence of in-depth quality assurance methods; interpretation by skilled, reliable, and independent observers; and validated tools for quantitative measurements.

In the United States, the cost of bringing a new drug to market is approaching $\$ 1$ billion, and requires several years of intense effort. Ways to expedite the approval process and reduce the cost of drug development would be most welcome by patients, healthcare providers, and payers. Imaging has the potential to improve this situation. However, for imaging to be most effective, the molecular mechanisms of the action of specific drugs should be better understood so that the right drugs can be developed for the right targets and for the right diseases.

\section{IMAGING OF CHRONIC METABOLIC DISEASE: DIABETES}

As effective methods have evolved to prevent and treat acute and infectious diseases, and as greater numbers of people are living to older ages, chronic diseases are becoming an increasing health and financial burden to society. Often these diseases are incurable, but their symptoms and consequences may be ameliorated by treatment. Two examples of chronic diseases in which biomedical imaging is useful in monitoring the effectiveness of treatment are asthma and diabetes mellitus. The latter is chosen to illustrate this role for biomedical imaging.

Type 2 diabetes mellitus is a serious health condition that is increasing dramatically in the United States. Currently, 21 million Americans suffer from diabetes and are at substantial risk for microvascular complications (e.g., eye, kidney, and nerve damage) and macrovascular complications that give rise to heightened morbidity and mortality from events such as coronary artery disease, myocardial infarction, stroke, congestive heart failure, and kidney failure. For example, $25 \%-40 \%$ of patients with congestive heart failure (CHF) have type 2 diabetes mellitus. The risk of $\mathrm{CHF}$ in diabetic patients is fourfold than in nondiabetic men and eightfold than in diabetic women. For diabetics, the lifetime risk of $\mathrm{CHF}$ is one in four, with $50 \%$ mortality within 2 years in symptomatic patients. Type 2 diabetes mellitus is a growing personal and public health issue that needs improved methods for prevention, diagnosis, treatment, monitoring, and follow-up. Imaging offers one highly-promising avenue to these improvements.

\section{IMAGING OF DIABETES: OPPORTUNITIES}

Imaging provides a noninvasive method for studying the mechanisms and complications of diabetes in humans and in genetically-created animal models, including the ability to track diabetes-induced changes in both over time. Imaging diabetic rodents reduces the cost, improves the understanding, and expedites better methods of controlling this chronic disease in humans.

Diabetes assaults the cardiovascular system, and cardiovascular imaging is an essential tool for understanding the biology and etiology of the disease. ${ }^{38-56}$ Imaging of chronic conditions associated with diabetes includes studies of atherosclerotic plaque formation, changes in fat distribution (e.g., visceral, myocardial), and glycosylation of tendons and other tissues. Imaging has the potential for early detection of type 2 diabetes and for monitoring the progression of the disease and the appearance of major risk factors for thrombosis, stenosis, and other complications. Imaging can aid in delineating vascular function (arterial distensibility and endothelial function) and in determining the effectiveness of treatments to stabilize or reverse progressive diabetesinduced deterioration of the vascular system.

Diabetes is a metabolic disorder that challenges the core knowledge of chemists, biologists, physicians, and behavioral scientists. Diabetes control and treatment, as well as reduction of the risk of its serious associated health effects, require a multidisciplinary team of these individuals, working with scientists and engineers to improve imaging methods and with biochemists and pharmacologists to develop qualitative and quantitative biomarkers to evaluate disease progression in humans and animals.

Use of imaging to gain an improved understanding of diabetes could lead to a better model of the disease, with more explicit biomarkers to evaluate disease progression in organs and in the peripheral vasculature. Included in this model would be influences such as age, gender, race, genetics, other chronic conditions, and the potential effects of previous acute illnesses. A more comprehensive model of diabetes, based on improved understanding of the disease and more useful diabetic animal models, would help reshape clinical research on diabetes and promote discovery of improved approaches to control and potentially cure type 2 diabetes mellitus, a chronic disease that is a major public health challenge for the nation.

Imaging for the detection of subclinical diabetes in asymptomatic individuals could lead to prevention or delay in the onset of the debilitating effects of diabetes, speed the development of new therapies and control measures, and substantially reduce the risk of life-threatening events associated with the disease. Further, imaging might eventually be used to monitor the progression of the disease and the effectiveness of therapeutic measures designed to slow disease progression.

\section{IMAGING OF DIABETES: CHALLENGES}

Diabetes is a chronic disease that is only incompletely understood, especially with regard to its cause, its relationship with cardiovascular disease, and the variability of its consequences in different individuals. Development of qualitative and quantitative biomarkers at all disease stages are needed to use imaging to improve the understanding of diabetes, monitor its progression in different organs and the pe- 
ripheral vasculature, and ascertain the effectiveness of treatment and control. Imaging methods on all scales, from molecular to whole animal and human, should be explored for their usefulness in gaining a firmer understanding of diabetes and its devastating effects on people.

Better animal models for diabetes are needed; a consortium similar to the mouse consortium of the National Cancer Institute would be helpful. On the technology side, improved imaging instrumentation for monitoring disease progression in the cardiovascular system is needed, as well as tools for image integration and analysis.

Understanding diabetes requires knowledge from many fields, including biochemistry, endocrinology, cardiovascular physiology, and molecular biology. Physicians who treat patients with diabetes need a core body of knowledge that encompasses all of these fields, making the endocrinology of diabetes one of the more challenging disciplines of medicine. Advances in diabetes treatment and management, and ultimately prevention, will require interdisciplinary teams from several specialties working together to improve the understanding of the causes and effects of the disease.

To explore the role of imaging in diabetes management and treatment, animal models are needed that more closely simulate diabetes in humans. Improved rodent imaging systems that provide improved spatial, contrast, and temporal resolution are also needed, as are standardized validation tools that facilitate the extrapolation of results in animals to improved management of diabetes in humans. Human imaging applications need better biomarkers that can be tracked using radiation doses that are within an acceptable range.

Diabetes should be declared an emerging healthcare crisis in the United States (and several other developed countries). Open forums should be created for discourse on the problem that include participants from the medical device industry, pharmaceutical companies, academics, and federal agencies. These forums should generate a communications network for information-sharing about the accuracy and reproducibility of imaging methods, the improvement of biomarkers and tracers, and results of efforts to improve the quantifiability of treatment outcomes and reduce the morbidity of diabetes. Finally, new scientists should be encouraged to enter the research arena of diabetes, which is a major challenge not only to afflicted individuals and to the health of the public, but also to the intellectual ability of scientists to master the multidisciplinary nature of the required research.

\section{FOUR-DIMENSIONAL IMAGE-GUIDED INTERVENTION}

Image-guided intervention (IGI) refers to the acquisition and manipulation of biomedical images to actively guide medical interventions. ${ }^{57-71}$ With the exceptions of ultrasound and $\mathrm{x}$-ray fluoroscopy, biomedical images tend to be static and therefore provide "snapshots" in time rather than continuous imaging through time. This limitation creates difficulties for image-guided intervention in regions of the body where voluntary and involuntary motion is present. Ways to overcome this limitation were the focus of the fourth plenary session of BIROW IV-Image-Guided Intervention in the fourth Dimension-Time (4D IGI). ${ }^{72}$

In this session, a major topic of discussion was the need to convene a multidisciplinary consensus group to begin the process of developing standards, sharing data, agreeing on validation methods, and networking individuals into collaborative research teams focused on 4D IGI. This network of research teams could work with federal agencies to develop standardized approaches to 4D IGI that would improve over time as a result of collaborative research among and across groups. (The research policy division of the Society for Interventional Radiology Foundation has initiated the development of a consortium of IGI Centers of Excellence.)

\section{D IGI: OPPORTUNITIES}

Improved methods are needed to quantify motion (e.g., respiratory, cardiac, peristalsis, iatrogenic) and its impact on organ deformation and the accuracy of IGI. These methods, which might include functional and molecular imaging techniques, would lead ultimately to better motion-correction algorithms and to more accurate ways to monitor disease response over time. As a consequence, the success (or lack thereof) of surgical interventions, radiation therapy, and ablative techniques should be more apparent and therefore more amenable to improvement.

4D IGI is highly technology-dependent and requires a multidisciplinary team of scientists, engineers, and physicians for its development and deployment. The need for this multidisciplinary approach led to a reiteration of the need for a mechanism to allow submission of research applications listing co-principal investigators as the individuals responsible for the research.

4D IGI is a rather recent technology, and its rate of development often outpaces the clinical validation of any particular technique. Not infrequently, a new 4D IGI technology obviates the need for a preceding one. A network of collaborative research teams would help address this challenge in three ways: expediting the clinical validation of a particular approach, facilitating collaboration among groups to improve research, and enhancing communication, so that research groups working in the field would know what others are doing.

Development of less invasive, more effective interventions is the ultimate goal of 4D IGI. These interventions could include individualized and targeted therapies monitored in real-time by molecular imaging techniques, employing new classes of traceable tools and instruments. Areas of diseased tissue that are less responsive to therapy may be identifiable with greater precision in the future, so that they can be treated through supplemental or alternative methods.

The objective of 4D IGI is safer and less-invasive interventions that yield greater success, which is ultimately the removal or healing of diseased tissue without damaging normal tissue to the degree that the patient's well-being is compromised. Another objective is the development and valida- 
tion of real-time treatment endpoints (biomarkers) to assess the degree to which patient outcome has been improved and patient discomfort has been minimized.

\section{D IGI: CHALLENGES}

Several key issues emerged during discussion of the challenges of 4D IGI. These issues include the need for: continued evolution of multiparametric imaging that encompasses anatomic, temporal, and functional information; real-time tracking of motion and organ deformation; automatic error identification methods that detect misregistration and "loss of target;" feedback mechanisms to automatically correct for misregistration and "loss of target;" improved ergonomics of interventional suites to accommodate imaging equipment and improve patient access; reducing or eliminating the exposure of patients to ionizing radiation during 4D IGI techniques; a benchmarking database in the public domain to facilitate research comparisons and collaboration through the use of predefined datasets.

4D-IGI is totally dependent on computer-generated data and images that must be provided as needed in the interventional suite. Consequently, 4D IGI must be an on-line process that provides accurate coregistration of data from different sources in real time.

New algorithms must be developed that meet this criterion within agreed-upon accuracy limits and time restrictions. Biomarkers of successful intervention must be identified for 4D IGI, that may be different from established end points. For example, successful treatment of a liver tumor by radio-frequency ablation can cause a temporary increase in the tumor diameter, and images of the post-treatment tumor would lead to the conclusion that the treatment failed according to the RECIST (Response Evaluation Criteria in Solid Tumors) criteria. [The RECIST criteria describe four categories of response of a tumor to drug therapy: CR (complete response)=disappearance of all target lesions; PR (partial response) $=30 \%$ decrease in the sum of the longest diameter of target lesions; PD (progressive disease) $=20 \%$ increase in the sum of the longest diameter of target lesions; SD (stable disease $)=$ small changes that do not meet the above criteria .

Technologies employed in 4D IGI are expensive, but the payoff is considerable in safer, less invasive, and more effective interventions. Fundamental research to improve 4D IGI is dependent on federal funding and requires the NIH, in particular, to heighten its receptivity to grant applications focused on technology development that may not be hypothesis-driven. In addition, investigators should realize the potential of research support from investors and industry as mechanisms to further the development of 4D IGI technologies.

Validating 4D IGI methods through comparison with conventional therapeutic approaches and with other IGI techniques is essential to deployment of the methods. Disseminating information about this validation to referring physicians and to healthcare regulators and payers is necessary to the adoption of 4D IGI methods.

\section{ACKNOWLEDGMENTS}

Linda B. Bresolin, Ph.D., Assistant Executive Director, Research and Education at the Radiological Society for North American, did an extraordinary job in editing this manuscript.

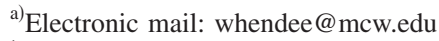

${ }^{1}$ P. L. Carson et al., "Biomedical Imaging Research Opportunities Workshop: Report and recommendations," Radiology 229, 328-339 (2003).

${ }^{2}$ C. L. Partain et al., "Biomedical Imaging Research Opportunities Workshop II: Report and recommendations," Radiology 236, 389-403 (2005). ${ }^{3}$ W. R. Hendee and G. S. Gazelle, "Biomedical Imaging Research Opportunities Workshop III: A white paper,” Ann. Biomed. Eng. 34, 188-198 (2006).

${ }^{4}$ E. Zerhouni, "Medicine. The NIH Roadmap," Science 302, 63-72 (2003).

${ }^{5}$ M. P. Kung and H. F. Kung, "Mass effect of injected dose in small rodent imaging by SPECT and PET," Nucl. Med. Biol. 32, 673-678 (2005).

${ }^{6}$ E. M. Jagoda et al., "Experiment assessment of mass effects in the rat: Implications for small animal PET imaging," Nucl. Med. Biol. 31, 771779 (2004).

${ }^{7}$ C. S. Levin, "Primer on molecular imaging technology," Eur. J. Nucl. Med. Mol. Imaging 32, Suppl. 2, S325-S345 (2005).

${ }^{8}$ A. Y. Nikitin et al., "Classification of proliferative pulmonary lesions of the mouse: Recommendations of the mouse models of human cancers consortium," Cancer Res. 64, 2307-2316 (2004).

${ }^{9}$ P. L. Chow et al., "A method of image registration for small animal, multimodality imaging," Phys. Med. Biol. 51, 379-390 (2006).

${ }^{10}$ A. J. Chaudhari et al., "Hyperspectral and multispectral bioluminescence optical tomography for small animal imaging," Phys. Med. Biol. 50, 5421-5441 (2005).

${ }^{11}$ S. Nikolaus et al., "State-of-the-art of small animal imaging with highresolution SPECT," Nuklearmedizin 44, 257-266 (2005).

${ }^{12} \mathrm{G}$. Zacharakis et al., "Fluorescent protein tomography scanner for small animal imaging," IEEE Trans. Med. Imaging 24, 878-885 (2005).

${ }^{13} \mathrm{P}$. Novak et al., "Experience with a small animal hyperthermia ultrasound system (SAHUS): Report on 83 tumours," Phys. Med. Biol. 50, 51275139 (2005).

${ }^{14}$ L. W. Dobrucki and A. J. Sinusas, "Molecular cardiovascular imaging," Curr. Cardiol. Rep. 7, 130-135 (2005).

${ }^{15} \mathrm{~S}$. K. Lyons, "Advances in imaging mouse tumour models in vivo," J. Pathol. 205, 194-205 (2005).

${ }^{16}$ M. Rodriguez-Porcel et al., "Image-guided cardiac cell delivery using high-resolution small-animal ultrasound," Mol. Ther. 12, 1142-1147 (2005).

${ }^{17}$ F. H. Fahey et al., "Evaluation of the quantitative capability of a highresolution positron emission tomography scanner for small animal imaging," J. Comput. Assist. Tomogr. 28, 842-848 (2004).

${ }^{18}$ E. E. Graves, R. Weissleder, and V. Ntziachristos, "Fluorescence molecular imaging of small animal tumor models," Curr. Mol. Med. 4, 419-430 (2004).

${ }^{19}$ M. L. Li et al., "A digital ultrasonic system for small animal imaging," Ultrason. Imaging 26, 85-99 (2004).

${ }^{20} \mathrm{Y}$. Yang et al., "Optimization and performance evaluation of the microPET II scanner for in vivo small-animal imaging," Phys. Med. Biol. 49, 2527-2545 (2004).

${ }^{21}$ B. A. Klaunberg and M. J. Lizak, "Considerations for setting up a smallanimal imaging facility," Lab Anim. 33, 28-34 (2004).

${ }^{22}$ H. Barthel, P. Price, and E. O. Aboagye, "Small-animal imaging of tumour proliferation with PET," Lancet 5, 100 (2004).

${ }^{23}$ J. M. Park and S. S. Gambhir, "Multimodality radionuclide, fluorescence and bioluminescence small-animal imaging," Proc. IEEE 93, 771-783 (2005).

${ }^{24}$ M. G. Pomper and J. S. Lee, "Small animal imaging in drug development," Curr. Pharm. Des. 11, 3247-3272 (2005).

${ }^{25}$ J. L. Tatum and J. M. Hoffman, "Congressional update: Report from the Biomedical Imaging Program of the National Cancer Institute. Imaging drug development," Acad. Radiol. 7, 1007-1008 (2000).

${ }^{26}$ A. Louie, "Design and characterization of magnetic resonance imaging gene reporters," Methods Mol. Med. 124, 401-417 (2006). 
${ }^{27}$ M. D. Silva and S. Chandra, "MRI in preclinical drug development," Methods Mol. Med. 124, 299-322 (2006).

${ }^{28}$ U. Haberkorn and M. Eisenhut, "Molecular imaging and therapy: A program based on the development of new biomolecules," Eur. J. Nucl. Med. Mol. Imaging 32, 1354-1359 (2005).

${ }^{29}$ W. C. Eckelman, J. A. Frank, and M. Brechbiel, "Theory and practice of imaging saturable binding sites," Invest. Radiol. 37, 101-106 (2002).

${ }^{30}$ W. C. Eckelman et al., "Are there lessons to be learned from drug development that will accelerate the use of molecular imaging probes in the clinic?," Nucl. Med. Biol. 32, 657-662 (2005).

${ }^{31}$ W. K. Schiffer et al., "Imaging addiction with PET: Is insight in sight?" Drug Discovery Today 10, 547-562 (2005).

${ }^{32} \mathrm{G}$. J. Kelloff et al., "The progress and promise of molecular imaging probes in oncologic drug development," Clin. Cancer Res. 11, 79677985 (2005).

${ }^{33} \mathrm{P}$. M. Smith-Jones et al., "Imaging the pharmacodynamics of HER2 degradation in response to Hsp90 inhibitors," Nat. Biotechnol. 22, 701-706 (2004).

${ }^{34}$ F. Sams-Dodd, "Target-based drug discovery: Is something wrong?" Drug Discovery Today 10, 139-147 (2005).

${ }^{35}$ B. J. Druker et al., "Efficacy and safety of a specific inhibitor of the BCR-ABL tyrosine kinase in chronic myeloid leukemia," N. Engl. J. Med. 344, 1031-1037 (2001).

${ }^{36}$ B. Ravina et al., "The role of radiotracer imaging in Parkinson disease," Neurology 64, 208-215 (2005).

${ }^{37} \mathrm{~K}$. A. Frey et al., "Presynaptic monoaminergic vesicles in Parkinson's disease and normal aging," Ann. Neurol. 40, 873-884 (1996).

${ }^{38}$ R. J. Genco et al., "A proposed model linking inflammation to obesity, diabetes, and periodontal infections," J. Periodontol. 76, Suppl. 11, 20752084 (2005).

${ }^{39}$ E. Reisin and M. A. Alpert, "Definition of the metabolic syndrome: Current proposals and controversies," Am. J. Med. Sci. 330, 269-272 (2005).

${ }^{40}$ L. Kappeler and J. Epelbaum, "Biological aspects of longevity and aging," Rev. Epidemiol. Sante Publique 53, 235-241 (2005).

${ }^{41}$ G. C. Fonarow, "An approach to heart failure and diabetes mellitus," Am. J. Cardiol. 96, 47E-52E (2005).

${ }^{42} \mathrm{C}$. M. Goeddeke-Merickel, "The goals of comprehensive and integrated disease state management for diabetic kidney-disease patients," Adv. Chronic Kidney Dis. 12, 236-242 (2005).

${ }^{43}$ M. Rijken et al., "Comorbidity of chronic diseases: Effects of disease pairs on physical and mental functioning," Qual. Life Res. 14, 45-55 (2005).

${ }^{44}$ S. Goya Wannamethee et al., "Overweight and obesity and the burden of disease and disability in elderly men," Int. J. Obes. Relat. Metab. Disord. 28, 1374-1382 (2004).

${ }^{45}$ S. M. Marshall, "Recent advances in diabetic nephropathy," Clin. Lab Med. 4, 277-282 (2004).

${ }^{46} \mathrm{Z}$. T. Bloomgarden, "Consequences of diabetes: Cardiovascular disease," Diabetes Care 27, 1825-1831 (2004).

${ }^{47}$ J. B. Meigs et al., "Biomarkers of endothelial dysfunction and risk of type 2 diabetes mellitus," J. Am. Med. Assoc. 291, 1978-1986 (2004)

${ }^{48} \mathrm{M}$. W. Stolar and R. J. Chilton, "Type 2 diabetes, cardiovascular risk, and the link to insulin resistance," Clin. Ther. 25, Suppl B, B4-B31 (2003).

${ }^{49} \mathrm{G}$. Paolisso et al., "Cardiovascular risk in type 2 diabetics and pharma- cological regulation of mealtime glucose excursions," Diabetes Metab. 29, 4 Pt 1, 335-340 (2003).

${ }^{50}$ V. Marks, "The metabolic syndrome," Nurs. Stand 17, 37-44 (2003).

${ }^{51}$ A. P. Harmel, "Treating diabetes: Cardiovascular benefits of antidiabetes drugs,” Am. J. Manag. Care 8, Suppl. 8, S219-S228 (2002); 8, Suppl. 8, quiz S229-S232 (2002).

${ }^{52} \mathrm{E}$. Ravussin and C. Bouchard, "Human genomics and obesity: Finding appropriate drug targets," Eur. J. Pharmacol. 410, 131-145 (2000).

${ }^{53} \mathrm{H}$. Keen, C. Clark, and M. Laakso, "Reducing the burden of diabetes: Managing cardiovascular disease," Diabetes/Metab. Rev. 15, 186-196 (1999).

${ }^{54}$ M. J. Franz, "Managing obesity in patients with comorbidities," J. Am. Diet Assoc. 98, 10 Suppl 2, S39-S43 (1998).

${ }^{55}$ R. N. Foley, P. S. Parfrey, and M. J. Sarnak, "Epidemiology of cardiovascular disease in chronic renal disease," J. Am. Soc. Nephrol. 9, Suppl. 12, S16-S23 (1998).

${ }^{56} \mathrm{E}$. Rossi, "Cardiovascular disease in diabetes and operative risk," Rays 22, 595-602 (1997).

${ }^{57}$ D. E. Dupuy, "Tumor ablation: Treatment and palliation using imageguided therapy," Oncology 19, 11 Suppl. 4, 4-5 (2005).

${ }^{58} \mathrm{M}$. Oldham et al., "Cone-beam-CT guided radiation therapy: A model for on-line application," Radiother. Oncol. 75, 271-278 (2005).

${ }^{59} \mathrm{~F}$. A. Jolesz et al., "MR imaging-controlled focused ultrasound ablation: A noninvasive image-guided surgery," Magn. Reson Imaging Clin. N. Am. 13, 545-560 (2005).

${ }^{60}$ E. J. Halpern, "Science to practice: High-intensity focused ultrasound ablation: Will image-guided therapy replace conventional surgery?" Radiology 235, 345-346 (2005).

${ }^{61}$ J. T. Yap et al., "Image-guided cancer therapy using PET/CT," Cancer J. 10, 221-233 (2004).

${ }^{62} \mathrm{D}$. Tomazevic et al., "3D/2D registration of CT and MR to x-ray images," IEEE Trans. Med. Imaging 22, 1407-1416 (2003).

${ }^{63}$ K. G. Vosburgh and F. A. Jolesz, "The concept of image-guided therapy," Acad. Radiol. 10, 176-179 (2003).

${ }^{64} \mathrm{P}$. Jannin et al., "Validation of medical image processing in image-guided therapy," IEEE Trans. Med. Imaging 21, 1445-1449 (2002).

${ }^{65}$ A. H. Chan et al., "An image-guided high intensity focused ultrasound device for uterine fibroids treatment," Med. Phys. 29, 2611-2620 (2002).

${ }^{66}$ D. Y. Chan et al., "Image-guided therapy in urology," J. Endourol 15, 105-110 (2001).

${ }^{67} \mathrm{~J}$. Kettenbach et al., "Interventional and intraoperative magnetic resonance imaging," Annu. Rev. Biomed. Eng. 2, 661-690 (2000).

${ }^{68}$ R. S. Hinks et al., "MR systems for image-guided therapy," J. Magn. Reson Imaging 8, 19-25 (1998).

${ }^{69} \mathrm{D}$. H. Gronemeyer et al., "Two- and three-dimensional imaging for interventional MRI and CT guidance," Stud. Health Technol. Inform. 29, 62-76 (1996).

${ }^{70}$ R. Leemuller, R. Bendl, and W. Schlegel, "Image-guided therapy planning for interventional stereotactic therapy of brain tumors," Radiologe 36, 737-743 (1996).

${ }^{71}$ M. W. Vannier and J. L. Marsh, "Three-dimensional imaging, surgical planning, and image-guided therapy," Radiol. Clin. North Am. 34, 545563 (1996).

${ }^{72}$ F. Laerum, "Demand for a new main speciality in image-guided therapy," Comput. Methods Programs Biomed. 66, 81-85 (2001). 